\title{
FLAVONOIDS AND CYANOGENIC GLYCOSIDES FROM THE LEAVES AND STEM BARK OF PRUNUS PERSICA (L.) BATSCH (MEET GHAMR) PEACH LOCAL CULTIVAR IN ASSIUT REGION
}

\author{
Enaam Y. Backheet, Salwa F. Farag, Amany S. Ahmed and Hanaa M. Sayed
}

Department of Pharmacognosy, Faculty of Pharmacy, Assiut University, Assiut, Egypt

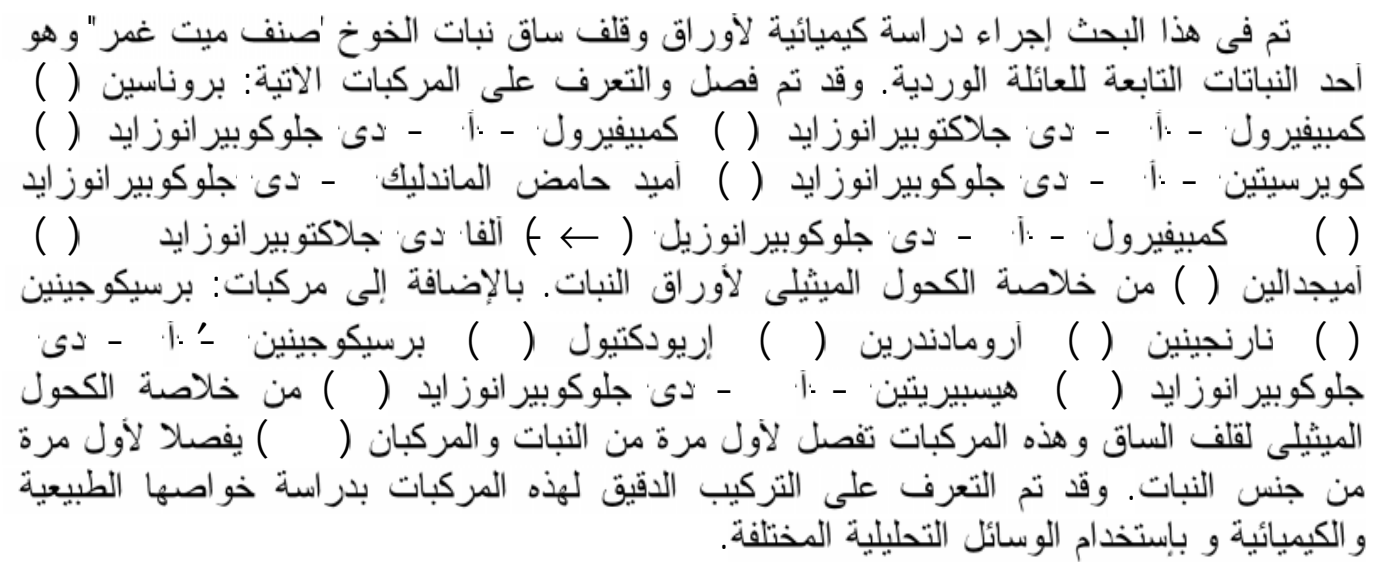

\begin{abstract}
Mandelonitrile- $\beta$-D-glucopyranoside (prunasin) (1), kaempferol- 3-O- $\beta$ - $D$ galactopyranoside (2), kaempferol-3-O- $\beta$-D-glucopyranoside (3), quercetin-3-O- $\beta$ - $D$ glucopyranoside (4), mandelic acid amide- $\beta$-D-glucopyranoside (5), kaempferol-3-O-[ $\beta$-Dglucopyranosyl-( $1 \rightarrow 4)$ - $\alpha$-D-galactopyranoside] (6), mandelonitrile- $\beta$ - $D$-glucopyranosyl$(1 \rightarrow 6)-\beta$-D-glucopyranoside (amygdalin) (7) were isolated from the methanolic extract of the leaves of Prunus persica (L.) Batsch "Meet Ghamr" peach. Furthermore, persicogenin (8), naringenin (9), aromadendrin (10), eriodictyol (11), persicogenin-3'-O- $\beta$-D-glucopyranoside (12) and hesperitin-5-O- $\beta$-glucopyranoside (13) were isolated from the methanolic extract of the stem bark of the title plant. All these compounds were isolated for the first time from "Meet Ghamr" peach while compounds 5 and $\mathbf{6}$ were firstly reported from the genus Prunus. Identification of these compounds has been established by physical, chemical and spectral methods (UV, IR, FAB-MS, 1-D-and 2-D NMR).
\end{abstract}

\section{INTRODUCTION}

Prunus persica (L.) Batsch, Family Rosaceae is a small bushy deciduous tree or large shrub with lanceolate tapering leaves and pink flowers appear in spring. ${ }^{1}$ The seeds or kernels, flowers and bark are used in medicine. The kernels are used as laxative, depurative, antispasmodic, for treatment of rheumatism, against cough and haemorrhages. Furthermore, they have been used to treat high blood pressure, blood diseases, colic and anemia. The flowers are diuretic and useful in dropsy and anuria. The inner white bark of the root is used as a prophylactic in epidemics and a remedy for dropsy and jaundice. It is quieting and insecticide. The immature leafy shoot with flowers and young fruit is cooked with pork and the broth ingested as a remedy for menstrual trouble, hemorrhage and hernia. A decoction of the leaves is used as a bath to treat heat rash, skin disease and circulatory troubles. ${ }^{2}$ Economically, the oil is used in skin creams; fruits are edible and used to flavour candy and ice-cream. ${ }^{1}$ Prunus persica extract may be useful for protection against UVinduced skin damage when topically applied. ${ }^{3}$ 
Several constituents have been isolated from the plants of genus Prunus such as triterpenes, ${ }^{4,5}$ phenyl propanoid glucose esters, ${ }^{6}$ lignan xylosides, ${ }^{7}$ flavans and proanthocyanidins, ${ }^{8,9}$ flavonols and anthocyanins, ${ }^{10}$ flavonoid-5-glucosides ${ }^{11}$ and phenolic glucosides. ${ }^{12,13}$

The previous phytochemical study of Prunus persica (L.) Batsch led to the isolation of persicogenin, multiflorin $\mathrm{A}$ and $\mathrm{B}$, multinoside A, chromogenic acid, quercetin, quercetrin, trifolin, astragalin, afzelin, gibberellin A-5, A-32, A-32 acetonide, GA-85, GA-86 and abscisic acid. ${ }^{3,14,15}$

The "Meet Ghamr" peach is the most important local variety in Egypt due to its adaptability to the environmental conditions. ${ }^{16}$ Upon reviewing the available literatures on the "Meet Ghamr" peach, nothing could be traced on its chemical constituents. Therefore, it was deemed of interest to carry a phytochemical study on this plant in order to evaluate the effect of environmental conditions on its chemical constituents. This study deals with the isolation of many flavonoids and cyanogenic glycosides from Prunus persica (L.) Batsch "Meet Ghamr" peach.

\section{EXPERIMENTAL}

\section{General experimental procedures}

1- Melting points are uncorrected and measured by Stuart Scientific melting point SMP1 instrument (England).

2- UV spectra were measured in methanol and different ionizing and complexing agents using Uvidec-320 spectrophotometer with matched $1 \mathrm{~cm}$ quartz cells (JASCO, Japan).

3- Schimadzu infra red-470 spectrometer (Japan) was used for measuring IR spectra in $\mathrm{KBr}$ discs.

4- 1-D and 2-D NMR spectra $\left({ }^{1} \mathrm{H}-{ }^{1} \mathrm{H}\right.$ COSY, HSQC and HMBC) were recorded on JEOL A-400, A-500 and A-600 spectrometers using TMS as an internal standard.

5- Positive FAB-MS spectra were recorded by JEOL HX-110 mass spectrometer (Japan) using glycerol or $m$-nitrobenzyl alcohol as a matrix.

6- The spots were visualized by UV lamp (254, 366 nm, VL, 6 LC, Marine Lavalee-
Cedex, France) and sprayed with $10 \%$ $\mathrm{H}_{2} \mathrm{SO}_{4}$ or $5 \% \mathrm{AlCl}_{3}$.

7- Column chromatography was performed with silica gel 60 (E. Merck), Develosil Lop ODS (30-50 $\mu$, Nomura chemicals) and sephadex LH-20 (Pharmacia Biotech. $\mathrm{AB}$, Upsala, Sweden).

8- Analytical TLC was conducted on precoated aluminium sheets of RP-18 $\mathrm{F}_{254} \mathrm{~S}$ (E. Merck) and silica gel $60 \mathrm{GF}_{254}$ (E. Merck).

9- Preparative TLC was performed on silica gel $60 \mathrm{GF}_{254}$ (E. Merck).

10- Authentic samples were obtained from Department of Pharmacognosy, Faculty of Pharmacy, Assiut University, Assiut.

11- Solvent systems:

A- Solvent systems were used for silica gel TLC:

I- Chloroform-methanol (90:10)

II- Ethyl acetate-ethanol (90:10)

III- Chloroform-methanol (70:30)

IV- $n$-Butanol-acetone-formic acidwater $(60: 17: 8: 15)$.

B- Solvent systems were used for RP-18 TLC:

water-methanol (20:10), (20:20) and $(20: 30)$

\section{Plant material}

Leaves and stem bark of Prunus persica (L.) Batsch "Meet Ghamr" peach were collected separately during the flowering stage (2001) from the plant cultivated in the Experimental Station, Faculty of Pharmacy, Assiut University, Assiut. The plant was identified by Prof. Dr. Samir El-Agamy, Department of Horticulture (Pomology), Faculty of Agriculture, Assiut University. The collected materials were air-dried, reduced to powder No. 40 and kept for extraction.

\section{Extraction and isolation I- Leaves}

The air-dried powdered leaves $(3.8 \mathrm{~kg})$ of Prunus persica (L.) Batsch "Meet Ghamr" peach were exhaustively extracted with methanol at room temperature and concentrated under vacuum. The concentrated extract (350 g) was diluted with distilled water and subjected to solvent fractionation using $n$ hexane $(6 \times 500 \mathrm{ml})$, chloroform $(5 \times 500 \mathrm{ml})$, ethyl acetate $(6 \times 500 \mathrm{ml})$ and $n$-butanol $(5 \times 500$ 
$\mathrm{ml})$. The obtained fractions were separately concentrated under reduced pressure till solvent-free residue $(200,40,50$ and $30 \mathrm{~g}$, respectively) and examined for different constituents by silica gel TLC using systems I and III.

\section{A- Ethyl acetate fraction}

About $15 \mathrm{~g}$ of the ethyl acetate soluble fraction was chromatographed on silica gel column $(450 \mathrm{~g}, 5 \times 150 \mathrm{~cm})$, and eluted with chloroform followed by chloroform-methanol gradient.Fractions of $250 \mathrm{ml}$ were collected, concentrated and monitored by silica gel TLC using systems I \& III. Five fractions were obtained; fraction I (1 g, eluted with chloroform), fraction II (5 g, eluted with chloroform-methanol 95:5), fraction III (4 g, eluted with chloroform-methanol 90:10), fraction IV (3.5 g, eluted with chloroformmethanol 85:15) and fraction $\mathrm{V}(1.3 \mathrm{~g}$, eluted with chloroform-methanol 80:20). About $3 \mathrm{~g}$ of fraction II was rechromatographed on ODS column (300 g, $5 \times 120 \mathrm{~cm})$ and eluted with water-methanol (30:10) to obtain compound $\mathbf{1}$ $(500 \mathrm{mg})$. Fraction III was rechromatographed on ODS column $(300 \mathrm{~g}, 5 \times 120 \mathrm{~cm})$, eluted with water-methanol (30:10) and (20:10) to yield compound 2 (300 mg) and compound 3 (200 $\mathrm{mg})$. Fraction IV was rechromatographed on silica gel column $(100 \mathrm{~g}, 2 \times 75 \mathrm{~cm})$ and eluted with chloroform-methanol (90:10) to afford compound 4 (200 mg).

\section{B- $\boldsymbol{n}$-Butanol fraction}

About $10 \mathrm{~g}$ of the $n$-butanol soluble fraction was fractionated on silica gel column $(300 \mathrm{~g}, 5 \times 120 \mathrm{~cm})$. Elution was started with ethyl acetate followed by ethyl acetatemethanol gradient. Fractions of $200 \mathrm{ml}$ were collected, concentrated and monitored by silica gel TLC using systems I \& III. Four fractions were obtained; fraction I ( $1 \mathrm{~g}$, eluted with ethyl acetate), fraction II (3 g, eluted with ethyl acetate-methanol 95:5), fraction III ( $2 \mathrm{~g}$, eluted with ethyl acetate-methanol 90:10) and fraction IV (3.8 g, eluted with ethyl acetate-methanol 80:20). Fraction II was rechromatographed on sephadex LH-20 using methanol. Further purification by preparative TLC using chloroform-methanol (80:20) afforded compound 5 (500 mg). Fraction III was rechromatographed on ODS column (300 g,
$5 \times 120 \mathrm{~cm})$ using water-methanol $(10: 20)$ to yield compound $6(50 \mathrm{mg})$. Fraction IV was purified on ODS column $(300 \mathrm{~g}, 5 \times 120 \mathrm{~cm})$ using water-methanol $(30: 10)$ to obtain compound 7 (40 mg).

\section{II- Stem bark}

The air-dried ground stem bark $(1.1 \mathrm{~kg})$ of Prunus persica (L.) Batsch "Meet Ghamr" peach was extracted with methanol at room temperature. The methanolic extract was concentrated under vacuum until solvent-free residue $(100 \mathrm{~g})$. The residue was diluted with distilled water and fractionated by using $n$ hexane $(3 \times 500 \mathrm{ml})$, chloroform $(3 \times 500 \mathrm{ml})$, ethyl acetate $(5 \times 500 \mathrm{ml})$ and $n$-butanol $(4 \times 500$ $\mathrm{ml})$. Each fraction was concentrated under reduced pressure to give the corresponding solubles $(15,10,50$ and $15 \mathrm{~g}$, respectively) and screened by silica gel TLC using system I.

\section{A- Chloroform fraction}

The chloroform soluble fraction $(10 \mathrm{~g})$ was chromatographed on silica gel column $(300 \mathrm{~g}, 5 \times 120 \mathrm{~cm})$ and elution was performed with $n$-hexane-acetone gradient. Fractions of $150 \mathrm{ml}$ were collected, concentrated and screened by silica gel TLC using system I. Three fractions were obtained; fraction I ( $2 \mathrm{~g}$, eluted with $n$-hexane-acetone 90:10), fraction II (4.8 g, eluted with $n$-hexane-acetone 80:20) and fraction III ( $3 \mathrm{~g}$, eluted with $n$-hexane-acetone 70:30). Fraction II was purified by repeated crystallization from methanol to obtain compound 8 (500 $\mathrm{mg})$. Fraction III was rechromatographed on silica gel column $(90 \mathrm{~g}$, $2 \times 75 \mathrm{~cm})$ and eluted with $n$-hexane-acetone (80:20) to yield compound $9(500 \mathrm{mg})$.

\section{B- Ethyl acetate fraction}

About $15 \mathrm{~g}$ of the ethyl acetate soluble fraction was fractionated on silica gel column $(450 \mathrm{~g}, 5 \times 150 \mathrm{~cm})$. Elution was started with chloroform followed by chloroform-methanol gradient. Fractions of $300 \mathrm{ml}$ were collected, concentrated under reduced pressure and monitored by silica gel TLC using system I. Similar fractions were combined to give five fractions; fraction I (800 mg, eluted with chloroform), fraction II (3 g, eluted with chloroform-methanol 95:5), fraction III (3.2 g, eluted with chloroform-methanol 90:10), fraction IV (4 g, eluted with chloroform- 
methanol 85:15) and fraction V (3.8 g, eluted with chloroform-methanol 80:20). About $2 \mathrm{~g}$ of each of fraction II and III was rechromatographed on ODS column (300 g, $5 \times 120 \mathrm{~cm})$ using water-methanol $(1: 1)$ to afford pure compounds $\mathbf{1 0}$ (70 mg) and 11 (100 $\mathrm{mg}$ ), respectively. Each of fraction IV and V was purified by repeated crystallization from methanol to yield compound $\mathbf{1 2}(300 \mathrm{mg})$ and compound $\mathbf{1 3}$ (200 mg), respectively.

\section{Acid hydrolysis}

Five mg portion of each of the isolated glycosides was dissolved in $5 \mathrm{ml}$ methanol to which $5 \mathrm{ml}$ of $\mathrm{N} / 2$ methanolic sulphuric acid was added. The mixture was refluxed for 3 hours on a water-bath and cooled. The aglycone was extracted with chloroform, purified and subjected to TLC. The produced sugars were identified by silica gel TLC using system IV.

\section{Compound 1}

White crystals [methanol], (500 mg), m.p $140-142^{\circ}, \mathrm{R}_{\mathrm{f}}=0.50$ (system II), FAB-MS at $m / z: 296[\mathrm{M}+1]^{+}$for $\mathrm{C}_{14} \mathrm{H}_{17} \mathrm{O}_{6} \mathrm{~N} .{ }^{1} \mathrm{H}-\mathrm{NMR}$ spectrum $\left(600 \mathrm{MHz}, \mathrm{DMSO}-d_{6}\right): \delta$ 3.07-3.10 $\left(4 \mathrm{H}, \mathrm{m}, \mathrm{H}-2^{\prime}, 3^{\prime}, 4^{\prime}, 5^{\prime}\right), 3.52(1 \mathrm{H}, \mathrm{dd}, J=5.50$, $\left.11.72 \mathrm{~Hz}, \mathrm{H}-6^{\prime} \mathrm{b}\right), 3.72$ (1H, dd, $J=6.60,11.72$ Hz, H-6'a), 4.22 (1H, d, J=7.30 Hz, H-1'), 6.03 (1H, s, H-7), 7.48 (3H, m, H-3, 4, 5), 7.57 (2H, $\mathrm{m}, \mathrm{H}-2,6) .{ }^{13} \mathrm{C}-\mathrm{NMR}$ spectrum $(150 \mathrm{MHz}$, DMSO- $\left.d_{6}\right): \delta 61.11\left(\mathrm{t}, \mathrm{C}-6^{\prime}\right), 66.59(\mathrm{~d}, \mathrm{C}-7)$, 69.87 (d, C-4'), 73.17 (d, C-2'), 76.51 (d, C-3'), 77.24 (d, C-5'), 101.15 (d, C-1'), 118.72 (s, CN), 127.35 (d, C-2,6), 128.95 (d, C-3,5), 129.60 (d, C-4), 133.69 (s, C-1).

\section{Compound 2}

Yellow amorphous powder, $(300 \mathrm{mg}), \mathrm{R}_{\mathrm{f}}=$ 0.50 (system III), UV ( $\left.\lambda_{\max }, \mathrm{nm} \mathrm{MeOH}\right): 265$, 289sh, 351; NaOMe: 275, 324sh, 401; $\mathrm{AlCl}_{3}$ : 273, 348, 395; $\mathrm{AlCl}_{3} / \mathrm{HCl}: 275,348,394$; NaOAc: 274, 305, 375: $\mathrm{NaOAc} / \mathrm{H}_{3} \mathrm{BO}_{3}$ : 265, 351. ${ }^{1} \mathrm{H}-\mathrm{NMR}$ spectrum (400 MHz, DMSO- $d_{6}$ ): $\delta$ 3.07-3.60 (sugar protons), $5.38(1 \mathrm{H}, \mathrm{d}, J=$ $\left.7.56 \mathrm{~Hz}, \mathrm{H}-1^{\prime \prime}\right), 6.17(1 \mathrm{H}, \mathrm{d}, J=2.00 \mathrm{~Hz}, \mathrm{H}-6)$, $6.40(1 \mathrm{H}, \mathrm{d}, J=2.00 \mathrm{~Hz}, \mathrm{H}-8), 6.84(2 \mathrm{H}, \mathrm{d}, J=$ $\left.8.00 \mathrm{~Hz}, \mathrm{H}-3^{\prime}, 5^{\prime}\right), 8.05(2 \mathrm{H}, \mathrm{d}, J=8.00 \mathrm{~Hz}, \mathrm{H}-$ $\left.2^{\prime}, 6^{\prime}\right), 12.59(1 \mathrm{H}, \mathrm{s}, 5-\mathrm{OH}) .{ }^{13} \mathrm{C}-\mathrm{NMR}(100$ MHz, DMSO- $\left.d_{6}\right)$ : $\delta 60.21$ (C-6"), 67.90 (C-4"),
71.24 (C-2"), 73.12 (C-3"), $75.81\left(\mathrm{C}-5^{\prime \prime}\right), 93.76$ (C-8), 98.82 (C-6), 101.72 (C-1"), 103.87 (C10), 115.15 (C-3',5'), 120.90 (C-1'), 131.07 (C2',6'), 133.24 (C-3), 156.34 (C-2), 156.44 (C9), 160.11 (C-5,4'), 161.23 (C-7), 177.53 (C-4).

\section{Compound 3}

Yellow amorphous powder, $(200 \mathrm{mg}), \mathrm{R}_{\mathrm{f}}=$ 0.59 (system III), UV ( $\left.\lambda_{\max }, \mathrm{nm} \mathrm{MeOH}\right): 265$, 300sh, 351; NaOMe: 273, 322sh, 404; $\mathrm{AlCl}_{3}$ : 275, 302sh, 346, 400; $\mathrm{AlCl}_{3} / \mathrm{HCl}: 275,348$, 403; NaOAc: 273, 371; $\mathrm{NaOAc} / \mathrm{H}_{3} \mathrm{BO}_{3}$ : 264, 351. ${ }^{1} \mathrm{H}-\mathrm{NMR}$ spectrum (400 MHz, DMSO- $\left.d_{6}\right)$ : $\delta$ 3.07-3.60 (sugar protons), $5.43(1 \mathrm{H}, \mathrm{d}, J=$ $\left.7.56 \mathrm{~Hz}, \mathrm{H}-1^{\prime \prime}\right), 6.17(1 \mathrm{H}, \mathrm{d}, J=2.00 \mathrm{~Hz}, \mathrm{H}-6)$, $6.39(1 \mathrm{H}, \mathrm{d}, J=2.00 \mathrm{~Hz}, \mathrm{H}-8), 6.87(2 \mathrm{H}, \mathrm{d}, J=$ $\left.8.00 \mathrm{~Hz}, \mathrm{H}-3^{\prime}, 5^{\prime}\right), 8.02(2 \mathrm{H}, \mathrm{d}, J=8.00 \mathrm{~Hz}, \mathrm{H}-$ $\left.2^{\prime}, 6^{\prime}\right), 12.56(1 \mathrm{H}, \mathrm{s}, 5-\mathrm{OH}) .{ }^{13} \mathrm{C}-\mathrm{NMR}(100$ MHz, DMSO- $\left.d_{6}\right): \delta 60.90$ (C-6"), 69.95 (C-4"), 74.29 (C-2"), 76.48 (C-3"), 77.54 (C-5"), 93.85 (C-8), 98.97 (C-6), 100.98 (C-1"), 103.81 (C10), 115.20 (C-3',5'), 120.98 (C-1'), 130.95 (C2', 6'), 133.21 (C-3), 156.23 (C-2), 156.54 (C9), 160.04 (C-4'), 161.26 (C-5), 164.99 (C-7), $177.44(\mathrm{C}-4)$.

\section{Compound 4}

Yellow amorphous powder, $(200 \mathrm{mg}), \mathrm{R}_{\mathrm{f}}=$ 0.44 (system III), UV ( $\left.\lambda_{\max }, \mathrm{nm} \mathrm{MeOH}\right): 257$, 269sh, 362; NaOMe: 272, 324sh, 409; $\mathrm{AlCl}_{3}$ : 273, 305sh, 438; $\mathrm{AlCl}_{3} / \mathrm{HCl}: 273,348,405$; NaOAc: 274, 324, 375; $\mathrm{NaOAc} / \mathrm{H}_{3} \mathrm{BO}_{3}$ : 265, 298, 377. ${ }^{1} \mathrm{H}-\mathrm{NMR}$ spectrum $(400 \mathrm{MHz}$, DMSO- $d_{6}$ ): $\delta$ 3.08-3.64 (sugar protons), 5.45 $\left(1 \mathrm{H}, \mathrm{d}, J=7.32 \mathrm{~Hz}, \mathrm{H}-1^{\prime \prime}\right), 6.19$ (1H, d, $J=1.68$ $\mathrm{Hz}, \mathrm{H}-6), 6.39$ (1H, d, $J=1.68 \mathrm{~Hz}, \mathrm{H}-8), 6.83$ $\left(1 \mathrm{H}, \mathrm{d}, J=8.48 \mathrm{~Hz}, \mathrm{H}-5^{\prime}\right), 7.53(1 \mathrm{H}, \mathrm{d}, J=2.2$ $\left.\mathrm{Hz}, \mathrm{H}-2^{\prime}\right), 7.57\left(1 \mathrm{H}, \mathrm{dd}, J=2.2,8.48 \mathrm{~Hz}, \mathrm{H}-6^{\prime}\right)$, $12.62(1 \mathrm{H}, \mathrm{s}, 5-\mathrm{OH}) .{ }^{13} \mathrm{C}-\mathrm{NMR}(100 \mathrm{MHz}$, DMSO- $\left.d_{6}\right): \delta 61.03$ (C-6"), 69.99 (C-4"), 74.17 (C-2"), 76.56 (C-3"), 77.62 (C-5"), 93.62 (C-8), 98.78 (C-6), 100.93 (C-1"), 104.02 (C-10), 115.29 (C-2'), 116.27 (C-5'), 121.24 (C-1'), 121.68 (C-6'), 133.38 (C-3), 144.89 (C-3'), 148.55 (C-4'), 156.25 (C-2) 156.41 (C-9), 161.30 (C-5), 164.31 (C-7), 177.50 (C-4).

\section{Compound 5}

White crystals [methanol], (500 mg), m.p $149-151^{\circ}, \mathrm{R}_{\mathrm{f}}=0.21$ (system II), FAB-MS at 
<smiles>[R]OC([R])c1ccccc1</smiles>

\begin{tabular}{cll} 
Compound & \multicolumn{1}{c}{$\mathrm{R}_{1}$} & \multicolumn{1}{c}{$\mathrm{R}_{2}$} \\
$\mathbf{1}$ & $\mathrm{CN}$ & glucose \\
$\mathbf{5}$ & $\mathrm{CONH}_{2}$ & glucose \\
$\mathbf{7}$ & $\mathrm{CN}$ & glucose-glucose
\end{tabular}<smiles>[R1]Oc1c(-c2ccc(O)c([R2])c2)oc2cc(O)cc(O)c2c1=O</smiles>

$\begin{array}{cll}\text { Compound } & \mathrm{R}_{1} & \mathrm{R}_{2} \\ \mathbf{2} & \text { galactose } & \mathrm{H} \\ \mathbf{3} & \text { glucose } & \mathrm{H} \\ \mathbf{4} & \text { glucose } & \mathrm{OH} \\ \mathbf{6} & \text { galactose-glucose } & \mathrm{H}\end{array}$<smiles>[R6]Oc1cc(O[R20])c2c(c1)OC(c1ccc(O[R6])c([R4])c1)C([R])C2=O</smiles>

$\begin{array}{clllll}\text { Compound } & \mathrm{R}_{1} & \mathrm{R}_{2} & \mathrm{R}_{3} & \mathrm{R}_{4} & \mathrm{R}_{5} \\ \mathbf{8} & \mathrm{H} & \mathrm{H} & \mathrm{CH}_{3} & \mathrm{OH} & \mathrm{CH}_{3} \\ \mathbf{9} & \mathrm{H} & \mathrm{H} & \mathrm{H} & \mathrm{H} & \mathrm{H} \\ \mathbf{1 0} & \mathrm{OH} & \mathrm{H} & \mathrm{H} & \mathrm{H} & \mathrm{H} \\ \mathbf{1 1} & \mathrm{H} & \mathrm{H} & \mathrm{H} & \mathrm{OH} & \mathrm{H} \\ \mathbf{1 2} & \mathrm{H} & \mathrm{H} & \mathrm{CH}_{3} & \mathrm{O} \text {-glucose } & \mathrm{CH}_{3} \\ \mathbf{1 3} & \mathrm{H} & \text { glucose } & \mathrm{H} & \mathrm{OH} & \mathrm{CH}_{3}\end{array}$


$m / z: 314[\mathrm{M}+1]^{+}$for $\mathrm{C}_{14} \mathrm{H}_{19} \mathrm{O}_{7} \mathrm{~N}$. IR (KBr), $v_{\max } \mathrm{cm}^{-1}: 3410,3300,2925,1671,1449$, 1424, 1075, 1022. ${ }^{1} \mathrm{H}-\mathrm{NMR}$ spectrum (600 MHz, DMSO- $\left.d_{6}\right): \delta 2.89(1 \mathrm{H}, \mathrm{ddd}, J=2.10$, 6.60, $\left.8.20 \mathrm{~Hz}, \mathrm{H}-5^{\prime}\right), 3.04$ (2H, m, H-3', 4'), $3.13\left(1 \mathrm{H}, \mathrm{dd}, J=7.70,9.50 \mathrm{~Hz}, \mathrm{H}-2^{\prime}\right), 3.42(1 \mathrm{H}$, dd, $\left.J=6.60,11.70 \mathrm{~Hz}, \mathrm{H}-6^{\prime} \mathrm{b}\right), 3.65(1 \mathrm{H}, \mathrm{dd}, J=$ 2.10, $\left.11.70 \mathrm{~Hz}, \mathrm{H}-6^{\prime} \mathrm{a}\right), 3.87(1 \mathrm{H}, \mathrm{d}, J=7.70 \mathrm{~Hz}$, H-1'), $5.16(1 \mathrm{H}, \mathrm{s}, \mathrm{H}-7), 7.35(3 \mathrm{H}, \mathrm{m}, \mathrm{H}-3,4$, 5), $7.45(2 \mathrm{H}, \mathrm{m}, \mathrm{H}-2,6) .{ }^{13} \mathrm{C}-\mathrm{NMR}$ spectrum $\left(150 \mathrm{MHz}, \quad \mathrm{DMSO}-d_{6}\right): \delta \quad 61.09$ (t, C-6'), 70.18 (d, C-4'), 73.47 (d, C-2'), 75.89 (d, C3'), 77.17 (d, C-7), 77.41 (d, C-5'), 98.62 (d, C-1'), 127.81 (d, C-2,6), 128.14 (d, C-4), 128.16 (d, C-3 5), 136.75 (s, C-1), 171.95 (s, $\underline{\mathrm{CO}}$.

\section{Compound 6}

Yellow amorphous powder, $(50 \mathrm{mg}), \mathrm{R}_{\mathrm{f}}=$ 0.34 (system III), UV ( $\left.\lambda_{\max }, \mathrm{nm} \mathrm{MeOH}\right): 267$, 289sh, 350; NaOMe: 271, 323sh, 398; $\mathrm{AlCl}_{3}$ : 274, 345, 395; $\mathrm{AlCl}_{3} / \mathrm{HCl}: 274,345,395$; NaOAc: 274, 352; $\mathrm{NaOAc} / \mathrm{H}_{3} \mathrm{BO}_{3}: 267,351$. FAB-MS at $m / z: 611[\mathrm{M}+1]^{+}$for $\mathrm{C}_{27} \mathrm{H}_{30} \mathrm{O}_{16}$. ${ }^{1} \mathrm{H}-\mathrm{NMR}\left(600 \mathrm{MHz}, \mathrm{DMSO}-d_{6}\right)$ : Table $1 .{ }^{13} \mathrm{C}-$ NMR (150 MHz, DMSO- $\left.d_{6}\right)$ : Table 1.

Table 1: NMR spectral data of compound 6 in DMSO- $d 6$.

\begin{tabular}{|c|c|c|c|}
\hline Position & ${ }^{13} \mathrm{C}$ & ${ }^{1} \mathrm{H}$ & HMBC \\
\hline 2 & $156.42 \mathrm{C}$ & & $2^{\prime}, 6^{\prime}$ \\
\hline 3 & $134.34 \mathrm{C}$ & & $1^{\prime \prime}$ \\
\hline 4 & $177.63 \mathrm{C}$ & & \\
\hline 5 & $164.33 \mathrm{C}$ & & 6 \\
\hline 6 & $98.16 \mathrm{CH}$ & $6.28(1 \mathrm{H}, \mathrm{d}, J=1.83)$ & $5,7,8,10$ \\
\hline 7 & $166.93 \mathrm{C}$ & & 6,8 \\
\hline 8 & $93.75 \mathrm{CH}$ & $6.50(1 \mathrm{H}, \mathrm{d}, J=1.83)$ & $6,7,9,10$ \\
\hline 9 & $156.42 \mathrm{C}$ & & 8 \\
\hline 10 & $104.00 \mathrm{C}$ & & 6,8 \\
\hline $1^{\prime}$ & $120.36 \mathrm{C}$ & & $3^{\prime}, 5^{\prime}$ \\
\hline $2^{\prime}, 6^{\prime}$ & $130.46 \mathrm{CH}$ & $7.76(2 \mathrm{H}, \mathrm{d}, J=8.75)$ & $2,4^{\prime}$ \\
\hline $3^{\prime}, 5^{\prime}$ & $115.42 \mathrm{CH}$ & $6.97(2 \mathrm{H}, \mathrm{d}, J=8.75)$ & $1^{\prime}$ \\
\hline $4^{\prime}$ & $157.15 \mathrm{C}$ & & $2^{\prime}, 6^{\prime}$ \\
\hline $1^{\prime \prime}$ & $101.84 \mathrm{CH}$ & $5.16(1 \mathrm{H}, \mathrm{d}, J=4.00)$ & $3,3^{\prime \prime}$ \\
\hline $2^{\prime \prime}$ & $69.63 \mathrm{CH}$ & $4.08(1 \mathrm{H}, \mathrm{m})$ & $4 "$ \\
\hline $3^{\prime \prime}$ & $68.91 \mathrm{CH}$ & $3.40(1 \mathrm{H}, \mathrm{m})$ & $1^{\prime \prime}, 4^{\prime \prime}$ \\
\hline $4^{\prime \prime}$ & $81.93 \mathrm{CH}$ & $3.40(1 \mathrm{H}, \mathrm{m})$ & $2^{\prime \prime}, 3^{\prime \prime}, 5^{\prime \prime}, 1^{\prime \prime \prime}$ \\
\hline $5^{\prime \prime}$ & $70.26 \mathrm{CH}$ & $3.74(1 \mathrm{H}, \mathrm{m})$ & $4 " \prime$ \\
\hline $6^{\prime \prime}$ & $60.80 * \mathrm{CH}_{2}$ & $\begin{array}{l}3.44(1 \mathrm{H}, \mathrm{m}) \\
3.61(1 \mathrm{H}, \mathrm{m}) \\
\end{array}$ & \\
\hline $1^{\prime \prime \prime}$ & $104.65 \mathrm{CH}$ & $4.31(1 \mathrm{H}, \mathrm{d}, J=8.0)$ & $4^{\prime \prime}, 2^{\prime \prime \prime}$ \\
\hline $2^{\prime \prime \prime}$ & $74.40 \mathrm{CH}$ & $3.00(1 \mathrm{H}, \mathrm{m})$ & 1"', $3^{\prime \prime \prime}$ \\
\hline $3^{\prime \prime \prime}$ & $76.89 \mathrm{CH}$ & $3.16(1 \mathrm{H}, \mathrm{m})$ & $2^{\prime \prime \prime}, 4^{\prime \prime \prime}$ \\
\hline $44^{\prime \prime \prime}$ & $69.74 \mathrm{CH}$ & $3.07(1 \mathrm{H}, \mathrm{m})$ & $3^{\prime \prime \prime}$ \\
\hline $5^{\prime \prime \prime}$ & $76.55 \mathrm{CH}$ & $3.44(1 \mathrm{H}, \mathrm{m})$ & \\
\hline $6^{\prime \prime \prime}$ & $60.91 * \mathrm{CH}_{2}$ & $\begin{array}{l}3.44(1 \mathrm{H}, \mathrm{m}) \\
3.61(1 \mathrm{H}, \mathrm{m}) \\
\end{array}$ & \\
\hline $5-\mathrm{OH}$ & & $12.59(1 \mathrm{H}, \mathrm{s})$ & \\
\hline
\end{tabular}

*The assignments may be interchangeable $J$ value in $\mathrm{Hz}$. 


\section{Compound 7}

White crystals [methanol], $(40 \mathrm{mg}), \mathrm{m} \cdot \mathrm{p}$ 223-226 ${ }^{\circ}, \mathrm{R}_{\mathrm{f}}=0.26$ (system III), FAB-MS at $m / z: 458[\mathrm{M}+1]^{+}$for $\mathrm{C}_{20} \mathrm{H}_{27} \mathrm{O}_{11} \mathrm{~N}$. ${ }^{1} \mathrm{H}-\mathrm{NMR}$ spectrum $\left(600 \mathrm{MHz}, \mathrm{DMSO}-d_{6}\right): \delta 3.00-4.04$ (sugar protons), $4.26\left(1 \mathrm{H}, \mathrm{d}, J=7.80 \mathrm{~Hz}, \mathrm{H}-1^{\prime \prime}\right)$, $4.42\left(1 \mathrm{H}, \mathrm{d}, J=7.80 \mathrm{~Hz}, \mathrm{H}-1^{\prime}\right), 5.99(1 \mathrm{H}, \mathrm{s}, \mathrm{H}-$ 7), 7.51 (3H, m, H-3,4,5), 7.58 (2H, m, H-2 6). ${ }^{13} \mathrm{C}-\mathrm{NMR}$ spectrum $\left(150 \mathrm{MHz}, \mathrm{DMSO}-d_{6}\right): \delta$ 61.04 (t, C-6"), 66.77 (d, C-7), 68.47(t, C-6'), 70.05 (d, C-4"), 70.08 (C-4'), 73.13 (C-2'), 73.73 (d, C-2'), 76.47 (d, C-3'), 76.54 (C-3", 5"), 76.74 (d, C-5'), 101.58 (d, C-1"), 103.68 (d, C-1'), 118.81 (s, $\underline{\mathrm{CN}}), 127.28$ (d, C-2,6), 128.93 (d, C-4), 129.09 (d, C-3,5), 133.86 (s, C-1).

\section{Compound 8}

Colourless needles [methanol], (500 mg), m.p $165-167^{\circ}, \mathrm{R}_{\mathrm{f}}=0.85$ (system I), UV ( $\lambda_{\max }$, nm MeOH): 285, 332; NaOMe: 286, 335; $\mathrm{AlCl}_{3}: 309,365 ; \mathrm{AlCl}_{3} / \mathrm{HCl}: 308,365 ; \mathrm{NaOAc}$ : 285, 332; $\mathrm{NaOAc} / \mathrm{H}_{3} \mathrm{BO}_{3}: 285,331$. FAB-MS at $m / z: 317[\mathrm{M}+1]^{+}$for $\mathrm{C}_{17} \mathrm{H}_{16} \mathrm{O}_{6} \cdot{ }^{1} \mathrm{H}-\mathrm{NMR}$ $\left(600 \mathrm{MHz}, \mathrm{DMSO}-d_{6}\right): \delta 2.78(1 \mathrm{H}, \mathrm{dd}, J=2.93$, $17.22 \mathrm{~Hz}, \mathrm{H}-3 e q), 3.07(1 \mathrm{H}, \mathrm{dd}, J=12.82$, $17.22 \mathrm{~Hz}, \mathrm{H}-3 \mathrm{ax}), 3.81\left(3 \mathrm{H}, \mathrm{s}, 4^{\prime}-\mathrm{OCH}_{3}\right), 3.91$ $\left(3 \mathrm{H}, \mathrm{s}, 7-\mathrm{OCH}_{3}\right), 5.32(1 \mathrm{H}, \mathrm{dd}, J=2.93,12.82$ $\mathrm{Hz}, \mathrm{H}-2), 6.05(1 \mathrm{H}, \mathrm{d}, J=2.20 \mathrm{~Hz}, \mathrm{H}-6), 6.07$ $(1 \mathrm{H}, \mathrm{d}, J=2.20 \mathrm{~Hz}, \mathrm{H}-8), 6.88(1 \mathrm{H}, \mathrm{d}, J=8.43$ $\left.\mathrm{Hz}, \mathrm{H}-5^{\prime}\right), 6.92(1 \mathrm{H}, \mathrm{dd}, J=2.20,8.43 \mathrm{~Hz}, \mathrm{H}-$ $\left.6^{\prime}\right), 7.04\left(1 \mathrm{H}, \mathrm{d}, J=2.20 \mathrm{~Hz}, \mathrm{H}-2^{\prime}\right), 12.00(1 \mathrm{H}$, s, 5-OH). ${ }^{13} \mathrm{C}-\mathrm{NMR}$ spectrum $(150 \mathrm{MHz}$, DMSO- $\left.d_{6}\right): \delta 43.28(\mathrm{t}, \mathrm{C}-3), 55.75\left(\mathrm{q}, 4^{\prime}-\right.$ $\left.\mathrm{OCH}_{3}\right), 56.15$ (q, 7- $\left.\mathrm{OCH}_{3}\right), 79.04$ (d, C-2), 94.32 (d, C-8), 95.20 (d, C-6), 103.24 (s, C-10), 110.78 (d, C-5'), 112.77 (d, C-2'), 118.22 (d, C6'), 131.67 (s, C-1'), 146.06 (s, C-3'), 147.09 (s, C-4'), 162.94 (s, C-9), 164.22 (s, C-5), 168.07 (s, C-7), 196.04 (s, C-4).

\section{Compound 9}

Yellow needles [methanol], (500 mg), m.p $225-227^{\circ}, \mathrm{R}_{\mathrm{f}}=0.51$ (system I), UV ( $\lambda_{\max }, \mathrm{nm}$ $\mathrm{MeOH}): 291,328 \mathrm{sh}$; NaOMe: 243, 325; $\mathrm{AlCl}_{3}$ : 310, 374; $\mathrm{AlCl}_{3} / \mathrm{HCl}: 310,371$; $\mathrm{NaOAc}: 284 \mathrm{sh}$, 322; $\mathrm{NaOAc} / \mathrm{H}_{3} \mathrm{BO}_{3}: 291,332 \mathrm{sh}$. FAB-MS at $m / z: 273[\mathrm{M}+1]^{+}$for $\mathrm{C}_{15} \mathrm{H}_{12} \mathrm{O}_{5} \cdot{ }^{1} \mathrm{H}-\mathrm{NMR}(600$ $\left.\mathrm{MHz}, \mathrm{DMSO}-d_{6}\right): \delta 2.68(1 \mathrm{H}, \mathrm{dd}, J=2.93$, $17.22 \mathrm{~Hz}, \mathrm{H}-3 e q), 3.26(1 \mathrm{H}, \mathrm{dd}, J=12.82$, $17.22 \mathrm{~Hz}, \mathrm{H}-3 \mathrm{ax}), 5.44(1 \mathrm{H}, \mathrm{dd}, J=2.93,12.82$ $\mathrm{Hz}, \mathrm{H}-2), 5.88$ (2H, br s, H-6, 8), $6.80(2 \mathrm{H}, \mathrm{d}$, $\left.J=8.79, \mathrm{H}-3^{\prime}, 5^{\prime}\right), 7.32\left(2 \mathrm{H}, \mathrm{d}, J=8.79, \mathrm{H}-2^{\prime}\right.$,
6'), $12.14(1 \mathrm{H}, \mathrm{s}, 5-\mathrm{OH}) .{ }^{13} \mathrm{C}-\mathrm{NMR}$ spectrum $\left(150 \mathrm{MHz}, \mathrm{DMSO}-d_{6}\right): \delta 41.93$ (t, C-3), 78.36 (d, C-2), 94.94 (d, C-8), 95.76 (d, C-6), 101.69 (s, C-10), 115.11 (d, C-3',5'), 128.24 (d, C-2', 6'), 128.82 (s, C-1'), 157.69 (s, C-4'), 162.88 (s, C-9), 163.44 (s, C-5), 166.69 (s, C-7), 196.25 (s, C-4).

\section{Compound 10}

Yellowish brown crystals [methanol], (70 mg), m.p 220-222 ${ }^{\circ}, R_{\mathrm{f}}=0.40$ (system I), UV $\left(\lambda_{\max }, \mathrm{nm} \mathrm{MeOH}\right): 290,327 \mathrm{sh}$; NaOMe: 245, $325 ; \mathrm{AlCl}_{3}: 274 \mathrm{sh}, 316,382 ; \mathrm{AlCl}_{3} / \mathrm{HCl}: 280 \mathrm{sh}$, 311, 378; NaOAc: 254, 284sh, 327; $\mathrm{NaOAc} / \mathrm{H}_{3} \mathrm{BO}_{3}: 295,336 \mathrm{sh}$. FAB-MS at $\mathrm{m} / \mathrm{z}$ : $289[\mathrm{M}+1]^{+}$for $\mathrm{C}_{15} \mathrm{H}_{12} \mathrm{O}_{6} \cdot{ }^{1} \mathrm{H}-\mathrm{NMR}(600 \mathrm{MHz}$, DMSO- $\left.d_{6}\right): \delta 4.58(1 \mathrm{H}, \mathrm{d}, J=11.36 \mathrm{~Hz}, \mathrm{H}-3)$, $5.06(1 \mathrm{H}, \mathrm{d}, J=11.36 \mathrm{~Hz}, \mathrm{H}-2), 5.86(1 \mathrm{H}, \mathrm{d}, J=$ $2.20 \mathrm{~Hz}, \mathrm{H}-8), 5.91(1 \mathrm{H}, \mathrm{d}, J=2.20 \mathrm{~Hz}, \mathrm{H}-6)$, $6.79\left(2 \mathrm{H}, \mathrm{d}, J=8.79 \mathrm{~Hz}, \mathrm{H}-3^{\prime}, 5^{\prime}\right), 7.31(2 \mathrm{H}, \mathrm{d}$, $\left.J=8.79, \mathrm{H}-2^{\prime}, 6^{\prime}\right), 11.90(1 \mathrm{H}, \mathrm{s}, 5-\mathrm{OH}) .{ }^{13} \mathrm{C}-$ NMR spectrum (150 MHz, DMSO- $\left.d_{6}\right): \delta 71.44$ (d, C-3), 82.86 (d, C-2), 95.00 (d, C-8), 96.02 (d, C-6), 100.41 (s, C-10), 114.89 (d, C-3',5'), 128.83 (s, C-1'), 129.41 (d, C-2', 6'), 157.71 (s, C-4'), 162.55 (s, C-9), 163.98 (s, C-5), 166.89 (s, C-7), 197.76 (s, C-4).

\section{Compound 11}

Pale yellow powder, $(100 \mathrm{mg}), \mathrm{R}_{\mathrm{f}}=0.37$ (system I), UV ( $\left.\lambda_{\max }, \mathrm{nm} \mathrm{MeOH}\right): 289,324 \mathrm{sh}$; NaOMe: 246, 322; $\mathrm{AlCl}_{3}: 307,378 ; \mathrm{AlCl}_{3} / \mathrm{HCl}$ : 307, 373; NaOAc: 289, 323; $\mathrm{NaOAc} / \mathrm{H}_{3} \mathrm{BO}_{3}$ : 290, 333sh. FAB-MS at $\mathrm{m} / \mathrm{z}: 289[\mathrm{M}+1]^{+}$for $\mathrm{C}_{15} \mathrm{H}_{12} \mathrm{O}_{6} \cdot{ }^{1} \mathrm{H}-\mathrm{NMR}\left(500 \mathrm{MHz}\right.$, DMSO- $\left.d_{6}\right): \delta$ $2.68(1 \mathrm{H}, \mathrm{dd}, J=2.75,16.95 \mathrm{~Hz}, \mathrm{H}-3 e q), 3.17$ $(1 \mathrm{H}, \mathrm{dd}, J=12.37,16.95 \mathrm{~Hz}, \mathrm{H}-3 a x), 5.38(1 \mathrm{H}$, $\mathrm{dd}, J=2.75,12.37 \mathrm{~Hz}, \mathrm{H}-2), 5.87(2 \mathrm{H}$, br s, H6, 8), $6.74\left(2 \mathrm{H}\right.$, br s, H-5', $\left.6^{\prime}\right), 6.87(1 \mathrm{H}$, br s, H$\left.2^{\prime}\right), 12.13(1 \mathrm{H}, \mathrm{s}, 5-\mathrm{OH}) .{ }^{13} \mathrm{C}-\mathrm{NMR}$ spectrum $\left(125 \mathrm{MHz}, \mathrm{DMSO}-d_{6}\right): \delta 42.03(\mathrm{t}, \mathrm{C}-3), 78.37$ (d, C-2), 94.94 (d, C-8), 95.73 (d, C-6), 101.69 (s, C-10), 114.27 (d, C-2'), 115.28 (d, C-5'), 117.86 (s, C-6'), 129.43 (s, C-1'), 145.14 (s, C3'), 145.65 (s, C-4'), 162.83 (s, C-9), 163.44 (s, C-5), 166.74 (s, C-7), 196.20 (s, C-4).

\section{Compound 12}

White powder, $(300 \mathrm{mg}), \quad \mathrm{R}_{\mathrm{f}}=0.26$ (system I), UV $\left(\lambda_{\max }, \mathrm{nm} \mathrm{MeOH}\right): 286,332$; NaOMe: 286, 334; $\mathrm{AlCl}_{3}: 308,365 ; \mathrm{AlCl}_{3} / \mathrm{HCl}$ : 308, 365; NaOAc: 285, 333; $\mathrm{NaOAc} / \mathrm{H}_{3} \mathrm{BO}_{3}$ : 
285, 333. FAB-MS at $m / z: 479[\mathrm{M}+1]^{+}$for $\mathrm{C}_{23} \mathrm{H}_{26} \mathrm{O}_{11}$. ${ }^{1} \mathrm{H}-\mathrm{NMR}$ (600 MHz, DMSO- $\left.d_{6}\right)$ : Table 2. ${ }^{13} \mathrm{C}-\mathrm{NMR}\left(150 \mathrm{MHz}, \mathrm{DMSO}-d_{6}\right)$ : Table 2.

\section{Compound 13}

White powder, $(200 \mathrm{mg}), \mathrm{R}_{\mathrm{f}}=0.10$ (system I), UV $\left(\lambda_{\max }, \mathrm{nm} \mathrm{MeOH}\right): 281,325$; NaOMe: 242,$325 ; \mathrm{AlCl}_{3}$ : 282, 326; $\mathrm{AlCl}_{3} / \mathrm{HCl}$ : 282, 326; NaOAc: 255,$322 ; \mathrm{NaOAc} / \mathrm{H}_{3} \mathrm{BO}_{3}$ : 281, 325. FAB-MS at $\mathrm{m} / \mathrm{z}: 465[\mathrm{M}+1]^{+}$for $\mathrm{C}_{22} \mathrm{H}_{24} \mathrm{O}_{11} \cdot{ }^{1} \mathrm{H}-\mathrm{NMR}$ (500 MHz, DMSO- $d_{6}$ ): $\delta$ $2.65(1 \mathrm{H}, \mathrm{dd}, J=3.21,16.95 \mathrm{~Hz}, \mathrm{H}-3 e q), 2.98$
$(1 \mathrm{H}, \mathrm{dd}, J=12.83,16.95 \mathrm{~Hz}, \mathrm{H}-3 a x), 3.21(1 \mathrm{H}$, $\left.\mathrm{t}, J=9.0 \mathrm{~Hz}, \mathrm{H}-4^{\prime \prime}\right), 3.29$ (2H, m, H-2", 3"), $3.34\left(1 \mathrm{H}\right.$, ddd, $\left.J=2.60,5.50,9.00 \mathrm{~Hz}, \mathrm{H}-5^{\prime \prime}\right)$, $3.54\left(1 \mathrm{H}, \mathrm{dd}, J=5.50,11.50 \mathrm{~Hz}, \mathrm{H}-66^{\prime \prime} \mathrm{b}\right), 3.74$ $\left(1 \mathrm{H}, \mathrm{dd}, J=2.60,11.50 \mathrm{~Hz}, \mathrm{H}-6{ }^{\prime \prime} \mathrm{a}\right), 3.78(3 \mathrm{H}, \mathrm{s}$, $\left.4^{\prime}-\mathrm{OCH}_{3}\right), 4.71\left(1 \mathrm{H}, \mathrm{d}, J=7.30 \mathrm{~Hz}, \mathrm{H}-1^{\prime \prime}\right), 5.37$ $(1 \mathrm{H}, \mathrm{dd}, J=3.21,12.83 \mathrm{~Hz}, \mathrm{H}-2), 6.10(1 \mathrm{H}, \mathrm{d}$, $J=2.29 \mathrm{~Hz}, \mathrm{H}-8), 6.41(1 \mathrm{H}, \mathrm{d}, J=2.29 \mathrm{~Hz}, \mathrm{H}-$ 6), $6.87\left(1 \mathrm{H}, \mathrm{dd}, J=2.29,8.71 \mathrm{~Hz}, \mathrm{H}-6^{\prime}\right), 6.91$ $\left(1 \mathrm{H}, \mathrm{d}, J=2.29 \mathrm{~Hz}, \mathrm{H}-2^{\prime}\right), 6.93(1 \mathrm{H}, \mathrm{d}, J=8.71$ $\left.\mathrm{Hz}, \quad \mathrm{H}-5^{\prime}\right), 9.06(1 \mathrm{H}, \quad$ s, $7-\mathrm{OH}) .{ }^{13} \mathrm{C}-\mathrm{NMR}$ spectrum $\left(125 \mathrm{MHz}, \mathrm{DMSO}-d_{6}\right): \delta 44.62$

Table 2: NMR spectral data of compound 12 in DMSO- $d_{6}$.

\begin{tabular}{|c|c|c|c|c|}
\hline Position & ${ }^{13} \mathrm{C}\left(\mathrm{lit}^{26}\right)$ & ${ }^{13} \mathrm{C}$ & ${ }^{1} \mathrm{H}$ & HMBC \\
\hline 2 & 79.2 & $78.55 \mathrm{CH}$ & $5.51(1 \mathrm{H}, \mathrm{dd}, J=2.75,12.83)$ & $3 a x, 2^{\prime}, 6^{\prime}$ \\
\hline $\begin{array}{l}3 e q \\
3 a x\end{array}$ & 41.9 & $41.92 \mathrm{CH}_{2}$ & $\begin{array}{l}2.76(1 \mathrm{H}, \mathrm{dd}, J=2.75,16.95) \\
3.34(1 \mathrm{H}, \mathrm{dd}, J=12.83,16.95)\end{array}$ & $\begin{array}{l}4 \\
2\end{array}$ \\
\hline 4 & 196.7 & $196.80 \mathrm{C}$ & & $3 e q$ \\
\hline 5 & 167.4 & $163.13 \mathrm{C}$ & & $5-\mathrm{OH}, 6$ \\
\hline 6 & 95.3 & $94.64 \mathrm{CH}$ & $6.09(1 \mathrm{H}, \mathrm{d}, J=2.29)$ & $\begin{array}{l}5-\mathrm{OH}, 5,7 \\
8,10\end{array}$ \\
\hline 7 & 146.2 & $167.41^{\mathrm{a}} \mathrm{C}$ & & $\begin{array}{l}6,8,7- \\
\mathrm{OCH}_{3} \\
\end{array}$ \\
\hline 8 & 93.7 & $93.82 \mathrm{CH}$ & $6.14(1 \mathrm{H}, \mathrm{d}, J=2.29)$ & $6,7,9,10$ \\
\hline 9 & 162.7 & $162.73 \mathrm{C}$ & & 8 \\
\hline 10 & 102.5 & $102.57 \mathrm{C}$ & & 6,8 \\
\hline $1^{\prime}$ & 120.5 & $130.64^{\mathrm{b}} \mathrm{C}$ & & $5^{\prime}$ \\
\hline $2^{\prime}$ & 113.7 & $113.84 \mathrm{CH}$ & $7.25(1 \mathrm{H}, \mathrm{d}, J=2.29)$ & $2,3^{\prime}, 4^{\prime}, 6^{\prime}$ \\
\hline $3^{\prime}$ & 163.1 & $146.31^{\mathrm{a}} \mathrm{C}$ & & $2^{\prime}, 5^{\prime}, 1^{\prime \prime}$ \\
\hline $4^{\prime}$ & 149.1 & $149.16 \mathrm{C}$ & & $\begin{array}{l}2^{\prime}, 5^{\prime}, 6^{\prime}, 4^{\prime}- \\
\mathrm{OCH}_{3}\end{array}$ \\
\hline $5^{\prime}$ & 112.1 & $112.21 \mathrm{CH}$ & $7.01(1 \mathrm{H}, \mathrm{d}, J=8.71)$ & $1^{\prime}, 3^{\prime}, 4^{\prime}$ \\
\hline $6^{\prime}$ & 130.6 & $120.57^{\mathrm{b}} \mathrm{CH}$ & $7.08(1 \mathrm{H}, \mathrm{dd}, J=2.29,8.71)$ & $2,2^{\prime}, 4^{\prime}$ \\
\hline $1^{\prime \prime}$ & 99.7 & $99.74 \mathrm{CH}$ & $4.95(1 \mathrm{H}, \mathrm{d}, J=7.33)$ & $3^{\prime}, 3^{\prime \prime}$ \\
\hline $2^{\prime \prime}$ & 73.0 & $73.12 \mathrm{CH}$ & $3.28(1 \mathrm{H}, \mathrm{m})$ & $3^{\prime \prime}, 4^{\prime \prime}$ \\
\hline $3 "$ & 76.9 & $76.97 \mathrm{CH}$ & $3.28(1 \mathrm{H}, \mathrm{m})$ & $4^{\prime \prime}, 1^{\prime \prime}$ \\
\hline $4^{\prime \prime}$ & 69.7 & $69.72 \mathrm{CH}$ & $3.15(1 \mathrm{H}, \mathrm{t}, J=9.00)$ & $3^{\prime \prime}, 5^{\prime \prime}$ \\
\hline $5 "$ & 76.9 & $77.01 \mathrm{CH}$ & $3.28(1 \mathrm{H}, \mathrm{m})$ & $4^{\prime \prime}, 6^{\prime \prime}$ \\
\hline $6^{\prime \prime}$ & 60.6 & $60.60 \mathrm{CH}_{2}$ & $\begin{array}{l}3.47(1 \mathrm{H}, \mathrm{dd}, J=6.42,12.37) \\
3.65(1 \mathrm{H}, \mathrm{dd}, J=1.83,12.37)\end{array}$ & $5^{\prime \prime}$ \\
\hline $4^{\prime}-\mathrm{OCH}_{3}$ & 55.8 & $55.85 \mathrm{CH}_{3}$ & $3.78(3 \mathrm{H}, \mathrm{s})$ & $4^{\prime}$ \\
\hline $7-\mathrm{OCH}_{3}$ & 55.6 & $55.71 \mathrm{CH}_{3}$ & $3.70(3 \mathrm{H}, \mathrm{s})$ & 7 \\
\hline $5-\mathrm{OH}$ & & & $12.01(1 \mathrm{H}, \mathrm{s})$ & 5,6 \\
\hline
\end{tabular}

${ }^{\mathrm{a}, \mathrm{b}}$ Revised assignments.

$J$ value in $\mathrm{Hz}$. 
(t, C-3), $55.69\left(\mathrm{q}, 4^{\prime}-\mathrm{OCH}_{3}\right), 60.71\left(\mathrm{t}, \mathrm{C}-6^{\prime \prime}\right)$, 69.63 (d, C-4"), 73.43 (d, C-2"), 75.59 (d, C3"), 77.52 (d, C-5"), 77.95 (d, C-2), 97.69 (d, C-8), 98.81 (d, C-6), 103.32 (d, C-1"), 105.45 (s, C-10), 112.02 (d, C-5'), 113.96 (d, C-2'), 117.55 (d, C-6'), 131.27 (s, C-1'), 146.44 (s, C3'), 147.80 (s, C-4'), 160.58 (s, C-5), 164.06 (s, C-9), 164.84 (s, C-7), 189.80 (s, C-4).

\section{RESULTS AND DISCUSSION}

The molecular formula for compound $\mathbf{1}$ was deduced as $\mathrm{C}_{14} \mathrm{H}_{17} \mathrm{O}_{6} \mathrm{~N}$ from FAB-MS, $m / z$ $296[\mathrm{M}+1]^{+}$. Its ${ }^{1} \mathrm{H}-\mathrm{NMR}$ spectrum showed signals at $\delta 7.48$ and 7.57 , representing a typical pattern for monosubstituted benzene ring and a sharp singlet signal at $\delta 6.03$ assigned for an oxygen bearing methine proton. Also, it showed a characteristic doublet for an anomeric proton at $\delta 4.22$ with high $J$ value $(J=7.33 \mathrm{~Hz})$ indicated the $\beta$-configuration of the glycosidic linkage. ${ }^{17}$ The ${ }^{13} \mathrm{C}$-NMR spectrum and DEPT experiment displayed signals at $\delta 61.11$ (d), 69.87 (d), 73.17 (d), 76.51 (d), 77.24 (d) and 101.15 (d) were corresponding to one $\beta$-glucopyranosyl moiety. ${ }^{17}$ In addition, four signals attributed to monosubstituted benzene ring appeared at $\delta$ 127.35 (d), 128.95 (d), 129.60 (d) and 133.69 (s). Also, they revealed one methine group at $\delta$ 66.59 (d) and a carbon signal at $\delta 118.72(q)$ of CN group. The HSQC spectral analysis of compound $\mathbf{1}$ assigned significantly the correlation between each carbon and its directly attached protons while the interpretation of the proton-proton couplings were established by measurements of ${ }^{1} \mathrm{H}-{ }^{1} \mathrm{H}$ COSY. The HMBC spectrum showed cross peaks between the proton signal at $\delta 6.03(\mathrm{H}-7)$ and the carbon signals at $\delta 118.72(\underline{\mathrm{CN}})$, $133.69(\mathrm{C}-1)$ and $127.35(\mathrm{C}-2,6)$. Also, the anomeric proton signal at $\delta 4.22$ showed longrange correlation with carbon signal at $\delta 66.59$ (C-7). The previous spectral data are in good agreement with those reported for mandelonitrile- $\beta$-D-glucopyranoside (prunasin). ${ }^{18,19}$

Compound 7, was assigned the molecular formula $\mathrm{C}_{20} \mathrm{H}_{27} \mathrm{O}_{11} \mathrm{~N}$ from its FAB-MS, $m / z 458$ $[\mathrm{M}+1]^{+}$. The ${ }^{1} \mathrm{H}-$ and ${ }^{13} \mathrm{C}-\mathrm{NMR}$ spectral data of compound $\mathbf{7}$ were very similar to those of compound 1 with an additional $\beta$ - glucopyranosyl moiety. This was confirmed by the existence of two anomeric signals at $\delta 4.42$ (d, $\left.J=7.80 \mathrm{~Hz}, \mathrm{H}-1^{\prime}\right), \delta 103.68$ (d, C-1') $\delta 4.26$ (d, $\left.J=7.80 \mathrm{~Hz}, \mathrm{H}-1^{\prime \prime}\right)$ and $\delta 101.58$ (d, C-1"), indicating its bioside nature. ${ }^{17}$ The downfield shift of $C^{-6} 6^{\prime}$ at $\delta \quad 68.47$ indicated the interglycosidic linkage to be $\left(1^{\prime \prime} \rightarrow 6^{\prime}\right) .^{17}$ The identity of the two sugars and their sequence were assigned by the ${ }^{1} \mathrm{H}-{ }^{1} \mathrm{H}$ COSY, HSQC and HMBC spectra. Compound 7 was concluded to be mandelonitrile- $\beta$-D-glucopyranosyl- $(1 \rightarrow 6)$ $\beta$-D- glucopyranoside (amygdalin) by comparison of its ${ }^{1} \mathrm{H}$ - and ${ }^{13} \mathrm{C}-\mathrm{NMR}$ spectral data with those reported. ${ }^{18,20}$ Prunasin and amygdalin were reported from the leaves of Prunus serotina and Prunus virginiana ${ }^{21}$ and this is the first report for their occurrence in the leaves of the title plant.

The FAB-MS of compound 5 showed $[\mathrm{M}+1]^{+}$at $\mathrm{m} / \mathrm{z} 314$ was consistent with the molecular formula $\mathrm{C}_{14} \mathrm{H}_{19} \mathrm{O}_{7} \mathrm{~N}$. The IR spectrum showed a strong amide absorption band at $1671 \mathrm{~cm}^{-1}$. The ${ }^{1} \mathrm{H}-\mathrm{NMR}$ spectrum was similar to that of compound $\mathbf{1}$ (prunasin). It showed signals at $\delta \quad 7.35$ and 7.45 corresponding to a monosubstituted aromatic ring, a methine proton at $\delta 5.16$ and an anomeric proton of one glucopyranosyl moiety at $\delta 3.87$ with a coupling constant $J_{1^{\prime}-2^{\prime}}$ of 7.70 $\mathrm{Hz}$, indicated the $\beta$-configuration. Its ${ }^{13} \mathrm{C}-\mathrm{NMR}$ spectrum exhibited the same pattern of compound 1 but contained a carbonyl resonance at $\delta 171.95$ instead of $\underline{\mathrm{CN}}$ resonance at $\delta$ 118.72. From the previous data and comparison with the reported data, ${ }^{19}$ compound 5 was identified as mandelic acid amide- $\beta$-Dglucopyranoside which was isolated for the first time from the genus Prunus. This compound can be considerd as the product of hydration of nitrile group of prunasin. ${ }^{19}$

The UV spectral data in methanol for compounds 2-4 indicated their nature as C-3 $\mathrm{OH}$ substituted flavonols. ${ }^{22}$ They were identified as kaempferol-3-O- $\beta$-Dgalactopyranoside (trifolin), kaempferol-3- $O-\beta$ $\mathrm{D}$-glucopyranoside (astragalin), and quercetin3-O- $\beta$-D-gluco-pyranoside by direct comparison of their spectral data with literature data $^{3,22-24}$. Acid hydrolysis followed by coTLC for each of the aglycone and sugar part with authentic samples confirmed their structures. 
FAB-MS of compound 6 showed $[\mathrm{M}+1]^{+}$ peak at $\mathrm{m} / \mathrm{z}, 611$ consistent with the molecular formula $\mathrm{C}_{27} \mathrm{H}_{30} \mathrm{O}_{16}$. The UV spectral data in methanol indicating its $\mathrm{C}-3 \mathrm{OH}$ substituted flavonol nature. ${ }^{22}$ Study of the effects of ionizing and complexing agents indicated the presence of free hydroxyl groups at C-5, C-7 and C-4'. Its ${ }^{1} \mathrm{H}-\mathrm{NMR}$ spectral data (Table 1) showed signals in the aromatic region at $\delta 6.28$ and 6.50 (each $1 \mathrm{H}, \mathrm{d}, J=1.83 \mathrm{~Hz}$ ) for $\mathrm{H}-6$ and H-8, respectively, another two doublets appeared at $\delta 6.97$ and 7.76 (each $2 \mathrm{H}, \mathrm{d}, J=$ $8.80 \mathrm{~Hz}$ ) for $\mathrm{H}-3^{\prime}, 5^{\prime}$ and $\mathrm{H}-2^{\prime}, 6^{\prime}$, respectively. In addition, two anomeric protons appeared as two doublets at $\delta 5.16\left(\mathrm{~J}=4.00 \mathrm{~Hz}, \mathrm{H}-1^{\prime \prime}\right)$ and $4.31\left(J=8.00 \mathrm{~Hz}, \mathrm{H}-1^{\prime \prime \prime}\right)$. The anomeric proton signal of the terminal sugar (H-1"') resonated upfield relative to that of the primary sugar $(\mathrm{H}-$ 1 "), indicating its $O$-sugar-sugar linkage, which support the suggestion of 3-diglycosylated kaempferol structure. ${ }^{23}$ The small coupling constant of the first anomeric signal indicated its $\alpha$-configuration and the high coupling constant of the second anomeric proton indicated the $\beta$-configuration. ${ }^{23}$ The ${ }^{13} \mathrm{C}-\mathrm{NMR}$ spectral data (Table 1) revealed carbon signals for kaempferol derivative ${ }^{24,25}$ and two anomeric signals at $\delta 101.84\left(\mathrm{~d}, \mathrm{C}-1^{\prime \prime}\right)$ and $\delta 104.65(\mathrm{~d}$, $\left.\mathrm{C}-1^{\prime \prime \prime}\right)$ together with 10 carbons in the region of sugars. The ${ }^{13} \mathrm{C}-\mathrm{NMR}$ chemical shifts of the two hexoses are consistent with those corresponding to one $\alpha$-D-galactopyranosyl and one $\beta$-D-glucopyranosyl moiety. ${ }^{17}$ The downfield shift of $\mathrm{C}-4$ " at $\delta 81.93$ suggesting that the interglycosidic linkage is $\beta-\mathrm{D}$ glucopyranosyl-(1"' $\left.\rightarrow 4^{\prime \prime}\right)-\alpha-D$ -

galactopyranoside. In the HMBC spectrum (Table 1), the two anomeric proton signals at $\delta$ 4.31 (H-1"') and $5.16\left(\mathrm{H}-1^{\prime \prime}\right)$ showed strong correlations with the carbon signals at $\delta 81.93$ (C-4") and 134.34 (C-3), respectively. Furthermore, a careful analysis of the ${ }^{1} \mathrm{H}-{ }^{1} \mathrm{H}$ COSY and HMBC spectra enabled the identification of all protons and carbons of the two sugars. From the above evidence, the structure of compound $\mathbf{6}$ was concluded to be kaempferol-3- $O$-[ $\beta$-D-glucopyranosyl- $(1 \rightarrow 4)$ $\alpha$-D-galactopyranoside] which was isolated for the first time from the genus Prunus.

Compound 8 was found to have the molecular formula $\mathrm{C}_{17} \mathrm{H}_{16} \mathrm{O}_{6}$ as deduced from FAB-MS, $m / z$ at $317[\mathrm{M}+1]^{+}$. The UV data and the study of the effect of ionizing and complexing agents on the UV absorption of compound $\mathbf{8}$ suggested a flavanone nucleus having a free hydroxyl group at C-5. ${ }^{22}$ The ${ }^{1} \mathrm{H}$ NMR spectrum established its structure as a flavanone derivative by the appearance of three aliphatic protons of ring $\mathrm{C}$ at $\delta 2.78(1 \mathrm{H}, \mathrm{dd}, J=$ 2.93, $17.22 \mathrm{~Hz}, \mathrm{H}-3 e q), 3.07$ (1H, dd, $J=12.82$, $17.22 \mathrm{~Hz}, \mathrm{H}-3 \mathrm{ax})$ and $5.32(1 \mathrm{H}, \mathrm{dd}, J=2.93$, $12.82 \mathrm{~Hz}, \mathrm{H}-2){ }^{23}$ Also, it displayed three aromatic protons of ring $\mathrm{B}$ with a characteristic ABX-type coupling at $\delta 6.88(1 \mathrm{H}, \mathrm{d}, J=8.43$ $\left.\mathrm{Hz}, \mathrm{H}-5^{\prime}\right), 6.92(1 \mathrm{H}, \mathrm{dd}, J=2.20,8.43 \mathrm{~Hz}, \mathrm{H}-$ $\left.6^{\prime}\right), 7.04\left(1 \mathrm{H}, \mathrm{d}, J=2.20 \mathrm{~Hz}, \mathrm{H}-2^{\prime}\right)$. In addition, one set of meta-coupled aromatic protons at $\delta$ $6.05(1 \mathrm{H}, \mathrm{d}, J=2.20 \mathrm{~Hz}, \mathrm{H}-6), 6.07$ (1H, d, $J=$ $2.20 \mathrm{~Hz}, \mathrm{H}-8)$ and two methoxyl groups at $\delta$ 3.80 and 3.91 (each $3 \mathrm{H}, \mathrm{s}$ ). The C-2 and C-3 shifts in the ${ }^{13} \mathrm{C}-\mathrm{NMR}$ spectrum, were in good accord with the reported values for flavanones. ${ }^{24,25}$ The HMBC spectrum showed cross peaks between $\mathrm{H}-2^{\prime}(\delta$ 7.04) and C-2, C$3^{\prime}, \mathrm{C}-4^{\prime}$ and C-6' $(\delta$ 79.04, 146.06, 147.09 and 118.22). Also, the two proton signals at $\delta 6.88$ $\left(\mathrm{H}-5^{\prime}\right)$ and $6.92\left(\mathrm{H}-6^{\prime}\right)$ showed cross peaks with

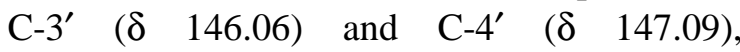
respectively. The signals of the methoxyl groups at $\delta 3.81$ and 3.91 showed distinct cross peaks with C-4' $(\delta$ 147.09) and C-7 ( $\delta$ 168.07), respectively. On the basis of these data, compound 8 was identified as 5,3'-dihydroxy$7,4^{\prime}$-dimethoxy flavanone (persicogenin).

The molecular formula of compound $\mathbf{1 2}$ was deduced as $\mathrm{C}_{23} \mathrm{H}_{26} \mathrm{O}_{11}$ from its FAB-MS, $\mathrm{m} / \mathrm{z}$ at $479[\mathrm{M}+1]^{+}$. Its ${ }^{1} \mathrm{H}-$ and ${ }^{13} \mathrm{C}-\mathrm{NMR}$ spectral data (Table 2) exhibited signals similar to compound $\mathbf{8}$ (persicogenin) and one $\beta$-Dglucopyranosyl moiety by the appearance of anomeric signals at $\delta 4.95(1 \mathrm{H}, \mathrm{d}, J=7.33 \mathrm{~Hz}$, $\left.\mathrm{H}-1^{\prime \prime}\right)$ and $\delta 99.74\left(\mathrm{~d}, \mathrm{C}-1^{\prime \prime}\right) .{ }^{17}$ All the carbons and their directly attached protons were well confirmed by the HSQC spectrum. Furthermore, acid hydrolysis of compound $\mathbf{1 2}$ gave glucose and persicogenin (by direct comparison with authentic samples, co-TLC and m.m.p). From these data, compound 12 could be identified as persicogenin $3^{\prime}-O-\beta-\mathrm{D}$ glucopyranoside. The study of HMBC spectrum of compound $\mathbf{1 2}$ led to revised assignments of the previously reported ${ }^{13} \mathrm{C}$ NMR resonances ${ }^{26}$ of C-7/C- $3^{\prime}$ and C-1'/C-6' (Table 2). 
Compounds 9-11 were identified as naringenin, dihydrokaempferol (aromadendrin) and eriodictyol, respectively by comparison of their spectral properties with literature data. ${ }^{22-25}$

FAB-MS of compound $\mathbf{1 3}$ showed $[\mathrm{M}+1]^{+}$ peak at $\mathrm{m} / z 465$ consistent with the molecular formula $\mathrm{C}_{22} \mathrm{H}_{24} \mathrm{O}_{11}$. The UV data showed characteristic absorption of flavanones. ${ }^{22}$ The study of the effect of ionizing and complexing agents suggested a free 7-OH group and substituted 5-OH group. ${ }^{22}$ The ${ }^{1} \mathrm{H}-\mathrm{NMR}$ spectrum exhibited one methoxy group at $\delta$ $3.78(3 \mathrm{H}, \mathrm{s})$ and protons of $\mathrm{C}-2$ and $\mathrm{C}-3$ of a flavanone at $\delta 2.65(1 \mathrm{H}, \mathrm{dd}, J=3.21,16.95 \mathrm{~Hz}$, $\mathrm{H}-3 e q), 2.98(1 \mathrm{H}, \mathrm{dd}, J=12.83,16.95 \mathrm{~Hz}, \mathrm{H}-$ $3 a x)$ and $5.37(1 \mathrm{H}, \mathrm{dd}, J=3.21,12.83 \mathrm{~Hz}, \mathrm{H}-$ $2) .{ }^{23}$ In addition, it displayed two aromatic meta-coupled protons of ring A at $\delta 6.10(\mathrm{H}-8)$ and $6.41(\mathrm{H}-6)($ each $1 \mathrm{H}, \mathrm{d}, J=2.29 \mathrm{~Hz})$ and three aromatic protons of ring $\mathrm{B}$ with an $\mathrm{ABX}$ type coupling at $\delta 6.87(1 \mathrm{H}, \mathrm{dd}, J=2.29,8.71$ $\left.\mathrm{Hz}, \mathrm{H}-6^{\prime}\right), 6.91\left(1 \mathrm{H}, \mathrm{d}, J=2.29 \mathrm{~Hz}, \mathrm{H}-2^{\prime}\right)$ and $\delta$ $6.93\left(1 \mathrm{H}, \mathrm{d}, J=8.71 \mathrm{~Hz}, \mathrm{H}-5^{\prime}\right)$. Furthermore, a characteristic doublet of an anomeric proton at $\delta 4.71(1 \mathrm{H}, \mathrm{d}, J=7.03 \mathrm{~Hz})$ indicated the presence of $\beta$-D-glucopyranosyl moiety. ${ }^{17,23}$ The ${ }^{13} \mathrm{C}$-NMR data revealed characteristic signals of flavanones at $\delta 44.62(\mathrm{t}, \mathrm{C}-3)$ and $77.95(\mathrm{~d}, \mathrm{C}-2)^{24,25}$ and an anomeric carbon of $\beta$ D-glucopyranosyl moiety ${ }^{17}$ at $\delta 103.32$ (d, C1"). The HSQC spectrum allowed the identification of all carbons and their protons. In the HMBC spectrum each of the proton signal at $\delta \quad 6.87 \quad\left(\mathrm{H}^{\prime} 6^{\prime}\right)$ showed strong correlations with carbon signals at $\delta 77.95$, 112.02 and 147.80 (C-2, C-2' and C-4'). Furthermore, the proton signal at $\delta 6.91\left(\mathrm{H}-2^{\prime}\right)$ showed cross peaks with three carbon signals at $\delta$ 77.95, 117.55, 131.27 and 147.80 (C-2, C-6', $\mathrm{C}-1^{\prime}$ and $\left.\mathrm{C}-4^{\prime}\right)$. Also, the proton signal at $\delta 6.93$ $\left(\mathrm{H}-5^{\prime}\right)$ was correlated with carbon signals at $\delta$ 131.27 and 146.44 (C-1', C-3'). In addition, the proton signal of methoxyl group was correlated with C-4' $(\delta 3.78 / 147.80)$. The presence of strong correlations between the proton signals at $\delta 4.71\left(\mathrm{H}-1^{\prime \prime}\right)$ and $6.41(\mathrm{H}-6)$ with the C-5 signal at $\delta 160.58$ confirmed the glycosidic linkage at position 5 of the aglycone. Consequently, compound $\mathbf{1 3}$ was identified as hesperitin 5-O- $\beta$-D-glucopyranoside.

In the course of the present work, it was observed that the flavonoids isolated from the leaves belong entirely to flavonols, while those isolated from the stem bark belong to flavanones and dihydroflavonols.

\section{Acknowledgments}

The authors wish to express their thanks to Prof. Dr. Masatake Niwa and Dr. Yoshiaki Takaya, Faculty of Pharmacy, Meijo University, Tempaku, Nagoya, Japan for carrying NMR and FAB mass spectra.

\section{REFERENCES}

1- D. Bown, "Encyclopedia of Herbs and their Uses", Dorling Kindersely Ltd., London, New York, Stuttgart, Moscow, p. 185 (1995).

2- L. M. Perry and J. Metzger, "Medicinal Plants of East and Southeast Asia, Attributed Properties and Uses", MIT Press, Cambridge, Massachusetts, London, England, p. 345 (1980).

3- Y. H. Kim, H. E.Yang, B. K. Park, M. Y. Heo, B. K. Jeo and H. P. Kim, J. Cosmet. Sci., 53, 27-34 (2002).

4- C. Fourneau, R. Hocquemiller and A. Cave, Phytochemistry, 42 (5), 1387-1389 (1996).

5- H. El Lahlou, N. Hirai, M.Tsuda and H.Ohigashi, Phytochemistry, 52 (4), 623630 (1999).

6- H. Shimomura, Y.Sashida and T. Adachi, Phytochemistry, 27 (2), 641-644 (1988).

7- K.Yoshinari, Y. Sashida and H. Shimomura, Chem. Pharm. Bull., 37 (12), 3301-3303 (1989).

8- M. S. M.Rawat, D. Prasad, R. K. Joshi and G. Pant, Phytochemistry, 50 (2), 321-324 (1999).

9- A. R. Bilia, I. Morelli, M. Hamburger and K. Hostettmann, Phytochemistry, 43 (4), 878-892 (1996).

10- F. A.Tomas-Barberan, M. Gil, P. Cremin, A. Waterhouse, B. Hers-Pierce and A. Kader, J. Agric. Food Chem., 49 (10), 4748-4760 (2001).

11- M. Geibel and W. Feucht, Phytochemistry, 30 (5), 1519-1521 (1991).

12- H. Shimomura, Y. Sashida and T. Adachi, Phytochemistry, 26 (1), 249-251 (1987).

13- O. M. Abdallah, M. S. Kamel and M. H. Mohamed, , Phytochemistry, 37 (6), 16891692 (1994). 
14- J. S. Glasby, "Dictionary of Plants Containing Secondary Metabolites", Taylor \& Francis, London, p. 263 (1991).

15- K. V.Bhaskar, W. L. A. Chu, P. A. Gaskin, L. N. Mander and N. Murofushi, Tetrahedron Lett., 32 (43), 6203-6206 (1991).

16- M. A. Hussein, K. M. Abdalla, H. M. Mahmoud and K. M. Farag, Assiut Journal of Agricultural Sciences, 14 (3), 359 (1983).

17- P. K. Agrawal, , Phytochemistry, 31 (10), 3307-3330 (1992).

18- W. Huebel, A. Nahrstedt and V. Wray, Arch. Pharm., 314 (7), 609-617 (1981).

19- A. Nahrstedt and J. Rockenbach, Phytochemistry, 34 (2), 433-436 (1993).

20- J. W. Turczan and T. Medwick, J. Assoc. Off. Anal. Chem., 62 (1), 190-196 (1979).
21- F. S. Santamour, Phytochemistry, 47 (8), 1537-1538 (1998).

22- T. J. Mabry, K. R. Markham and M. B. Thomas, "The Systematic Identification of Flavonoids“, Springer-Verlag, New York, Heidelberg, Berlin (1970).

23- J. B. Harborne, "The Flavonoids: Advances in Research Since 1986", Chapman \& Hall, London, Glasgow, New York, Tokyo, Melbourne, Madras (1994).

24- P. K. Agrawal, "Carbon-13 NMR of Flavonoids", Elsevier Science Ltd., Amsterdam, Oxford, New York, Tokyo (1989).

25- J. B. Harborne and T. J. Mabry, "The Flavonoids: Advances in Research", Chapman \& Hall, London (1982).

26- M. S. M.Rawat, G. Pant, R. Kukreti, J. Sakakibara and A. Nagatsu, Phytochemistry, 38 (6), 1519-1520 (1995). 